\title{
Momentum-space description of three-nucleon breakup reactions including the Coulomb interaction
}

\author{
A. Deltuva, ${ }^{1, *}$ A. C. Fonseca, ${ }^{1}$ and P. U. Sauer ${ }^{2}$ \\ ${ }^{1}$ Centro de Física Nuclear da Universidade de Lisboa, P-1649-003 Lisboa, Portugal \\ ${ }^{2}$ Institut für Theoretische Physik, Universität Hannover, D-30167 Hannover, Germany \\ (Received 4 August 2005; published 9 November 2005; publisher error corrected 22 November 2005)
}

\begin{abstract}
The Coulomb interaction between two protons is included in the calculation of proton-deuteron breakup and of three-body electromagnetic disintegration of ${ }^{3} \mathrm{He}$. The hadron dynamics is based on the purely nucleonic charge-dependent (CD) Bonn potential and its realistic extension, CD Bonn $+\Delta$, to a coupled-channel twobaryon potential, allowing for single virtual $\Delta$-isobar excitation. Calculations are done using integral equations in momentum space. The screening and renormalization approach is employed for including the Coulomb interaction. Convergence of the procedure is found at moderate screening radii. The reliability of the method is demonstrated. The Coulomb effect on breakup observables is seen at all energies in particular kinematic regimes.
\end{abstract}

DOI: 10.1103/PhysRevC.72.054004 PACS number(s): 21.30.-x, 21.45.+v, 24.70.+s, 25.10.+s

\section{INTRODUCTION}

The inclusion of the Coulomb interaction in the description of the three-nucleon continuum is one of the most challenging tasks in theoretical few-body nuclear physics [1]. Whereas it has already been solved for elastic proton-deuteron $(p d)$ scattering with realistic hadronic interactions by use of various procedures [1-5], there are only very few attempts [6-8] that have been made to calculate $p d$ breakup, and none of them uses a complete treatment of the Coulomb interaction and realistic hadronic potentials that allows for a stringent comparison with the experimental data.

Recently in Ref. [5] we included the Coulomb interaction between the protons in the description of three-nucleon reactions with two-body initial and final states. The description is based on the Alt-Grassberger-Sandhas (AGS) equation [9] in momentum space. The Coulomb potential is screened, and the resulting scattering amplitudes are corrected by the renormalization technique of Refs. $[10,11]$ to recover the unscreened limit. The treatment is applicable to any two-nucleon potential without separable expansion. Reference [5] and this paper use the purely nucleonic charge-dependent (CD) Bonn potential [12] and its coupled-channel extension, CD Bonn + $\Delta$ [13], allowing for a single virtual $\Delta$-isobar excitation and fitted to the experimental data with the same degree of accuracy as the CD Bonn itself. In the three-nucleon system the $\Delta$ isobar mediates an effective three-nucleon force and effective two- and three-nucleon currents, both consistent with the underlying two-nucleon force. The treatment of Ref. [5] is technically highly successful, but still limited to the description of $p d$ elastic scattering and of electromagnetic (e.m.) reactions involving ${ }^{3} \mathrm{He}$ with $p d$ initial or final states only. This paper extends the treatment of Coulomb to the breakup in $p d$ scattering and to the e.m. three-body disintegration of ${ }^{3} \mathrm{He}$. In

\footnotetext{
*Electronic address: deltuva@cii.fc.ul.pt; on leave from the Institute of Theoretical Physics and Astronomy, Vilnius University, Vilnius LT-2600, Lithuania.
}

that extension we follow the ideas of Refs. $[6,10,11]$ but avoid approximations on the hadronic potential and in the treatment of screened Coulomb. Thus, our three-particle equations, including the screened Coulomb potential, are completely different from the quasiparticle equations solved in Ref. [6], in which the two-nucleon screened Coulomb transition matrix is approximated by the screened Coulomb potential. In Ref. [14] we presented for the first time a limited set of results for $p d$ breakup using the same technical developments we explain here in greater detail.

We have to recall that the screened Coulomb potential $w_{R}$ we work with is particular. It is screened around the separation $r=R$ between two charged baryons and in configuration space is given by

$$
w_{R}(r)=w(r) e^{-(r / R)^{n}},
$$

with the true Coulomb potential $w(r)=\alpha_{e} / r, \alpha_{e}$ being the fine-structure constant and $n$ controlling the smoothness of the screening. We prefer to work with a sharper screening than the Yukawa screening $(n=1)$ of Ref. [6]. We want to ensure that the screened Coulomb potential $w_{R}$ approximates well the true Coulomb one $w$ for distances $r<R$ and simultaneously vanishes rapidly for $r>R$, providing a comparatively fast convergence of the partial-wave expansion. In contrast, the sharp cutoff $(n \rightarrow \infty)$ yields an unpleasant oscillatory behavior in the momentum-space representation, leading to convergence problems. We find the values $3 \leqslant n \leqslant 6$ to provide a sufficiently smooth, but at the same time a sufficiently rapid screening around $r=R$ as in Ref. [5]; $n=4$ is our choice for the results of this paper. The screening radius $R$ is chosen much larger than the range of the strong interaction that is of the order of the pion wavelength $\hbar / m_{\pi} c \approx 1.4 \mathrm{fm}$. Nevertheless, the screened Coulomb potential $w_{R}$ is of shortrange in the sense of scattering theory. Standard scattering theory is therefore applicable. A reliable technique [15] for solving the AGS equation [9] with short-range interactions is extended in Ref. [5] to include the screened Coulomb potential between the charged baryons. However, the partial-wave 
expansion of the pair interaction requires much higher angular momenta than the one of the strong two-nucleon potential alone.

The screening radius $R$ will always remain very small compared with nuclear screening distances of atomic scale, i.e., $10^{5} \mathrm{fm}$. Thus the employed screened Coulomb potential $w_{R}$ is unable to simulate properly the physics of nuclear screening and, even more, all features of the true Coulomb potential. Thus the breakup calculations with screened Coulomb $w_{R}$ have to be corrected for their shortcomings in a controlled way. References $[10,11]$ give the prescription for the correction procedure that we follow here for breakup as we did previously for elastic scattering, and that involves the renormalization of the on-shell amplitudes in order to get the proper unscreened Coulomb limit. After the indicated corrections, the predictions for breakup observables have to show independence from the choice of the screening radius $R$, provided it is chosen sufficiently large. That convergence is the internal criterion for the reliability of our Coulomb treatment.

Configuration space treatments of Coulomb [7,8] may provide a feasible alternative to the integral equation approach in momentum space on which this paper is based. References $[7,8]$ have provided first results for $p d$ breakup, but they still involve approximations in the treatment of Coulomb and the employed hadronic dynamics is not realistic. Thus a benchmark comparison between our breakup results and corresponding configuration space results is, in contrast to $p d$ elastic scattering [16], not possible yet. With respect to the reliability of our Coulomb treatment for breakup, we rely solely on our internal criterion, i.e., the convergence of breakup observables with the screening radius $R$; however, that criterion was absolutely reliable for $p d$ elastic scattering and related e.m. reactions.

Section II develops the technical apparatus underlying the calculations. Section III presents some characteristic effects of Coulomb in three-nucleon breakup reactions. Section IV gives our conclusions.

\section{TREATMENT OF COULOMB INTERACTION BETWEEN PROTONS IN BREAKUP}

This section carries over the treatment of the Coulomb interaction given in Ref. [5] for $p d$ elastic scattering and corresponding e.m. reactions, to $p d$ breakup and to e.m. three-body disintegration of ${ }^{3} \mathrm{He}$. It establishes a theoretical procedure leading to a calculational scheme. The discussions of hadronic and e.m. reactions are done separately.

\section{A. Theoretical framework for the description of proton-deuteron breakup with Coulomb}

This subsection focuses on $p d$ breakup. However, the transition matrices for elastic scattering and breakup are so closely connected that certain relations between scattering operators already developed in Ref. [5] have to be recalled to make this paper self-contained.

Each pair of nucleons $(\beta \gamma)$ interacts through the strong coupled-channel potential $v_{\alpha}$ and the Coulomb potential $w_{\alpha}$.
We assume that $w_{\alpha}$ acts formally between all pairs $(\beta \gamma)$ of particles, but it is nonzero only for states with two-charged baryons, i.e., $p p$ and $p \Delta^{+}$states. We introduce the full resolvent $G^{(R)}(Z)$ for the auxiliary situation in which the Coulomb potential $w_{\alpha}$ is screened with a screening radius $R, w_{\alpha}$ being replaced with $w_{\alpha R}$ :

$$
G^{(R)}(Z)=\left(Z-H_{0}-\sum_{\sigma} v_{\sigma}-\sum_{\sigma} w_{\sigma R}\right)^{-1}
$$

where $H_{0}$ is the three-particle kinetic-energy operator. The full resolvent yields the full $p d$ scattering state when acting on the initial channel state $\left|\phi_{\alpha}\left(\mathbf{q}_{i}\right) v_{\alpha_{i}}\right\rangle$ of relative $p d$ momentum $\mathbf{q}_{i}$, energy $E_{\alpha}\left(q_{i}\right)$, and additional discrete quantum numbers $v_{\alpha_{i}}$ and taking the appropriate limit $Z=E_{\alpha}\left(q_{i}\right)+i 0$. The full $p d$ scattering state has, above breakup threshold, components corresponding to the final breakup channel states $\left|\phi_{0}\left(\mathbf{p}_{f} \mathbf{q}_{f}\right) v_{0_{f}}\right\rangle, \mathbf{p}_{f}$ and $\mathbf{q}_{f}$ being three-nucleon Jacobi momenta, $E_{0}\left(p_{f} q_{f}\right)$ its energy, and $v_{0_{f}}$ additional discrete quantum numbers. The full resolvent therefore also yields the desired $S$ matrix for breakup. The full resolvent $G^{(R)}(Z)$ depends on the screening radius $R$ for the Coulomb potential, and that dependence is notationally indicated; the same will be done for operators related to $G^{(R)}(Z)$. Following standard AGS notation [9] of three-particle scattering, one may decompose the full resolvent $G^{(R)}(Z)$ into channel resolvents and free resolvent,

$$
\begin{aligned}
G_{\alpha}^{(R)}(Z) & =\left(Z-H_{0}-v_{\alpha}-w_{\alpha R}\right)^{-1}, \\
G_{0}(Z) & =\left(Z-H_{0}\right)^{-1},
\end{aligned}
$$

together with the full multichannel three-particle transition matrices $U_{\beta \alpha}^{(R)}(Z)$ for elastic scattering and $U_{0 \alpha}^{(R)}(Z)$ for breakup according to

$$
\begin{aligned}
& G^{(R)}(Z)=\delta_{\beta \alpha} G_{\alpha}^{(R)}(Z)+G_{\beta}^{(R)}(Z) U_{\beta \alpha}^{(R)}(Z) G_{\alpha}^{(R)}(Z), \\
& G^{(R)}(Z)=G_{0}(Z) U_{0 \alpha}^{(R)}(Z) G_{\alpha}^{(R)}(Z) .
\end{aligned}
$$

The full multichannel transition matrices satisfy the AGS equations [9]:

$$
\begin{aligned}
& U_{\beta \alpha}^{(R)}(Z)=\bar{\delta}_{\beta \alpha} G_{0}^{-1}(Z)+\sum_{\sigma} \bar{\delta}_{\beta \sigma} T_{\sigma}^{(R)}(Z) G_{0}(Z) U_{\sigma \alpha}^{(R)}(Z), \\
& U_{0 \alpha}^{(R)}(Z)=G_{0}^{-1}(Z)+\sum_{\sigma} T_{\sigma}^{(R)}(Z) G_{0}(Z) U_{\sigma \alpha}^{(R)}(Z),
\end{aligned}
$$

with $\bar{\delta}_{\beta \alpha}=1-\delta_{\beta \alpha}$; the two-particle transition matrix $T_{\alpha}^{(R)}(Z)$ is derived from the full channel interaction $v_{\alpha}+w_{\alpha R}$ including the screened Coulomb, i.e.,

$$
T_{\alpha}^{(R)}(Z)=\left(v_{\alpha}+w_{\alpha R}\right)+\left(v_{\alpha}+w_{\alpha R}\right) G_{0}(Z) T_{\alpha}^{(R)}(Z) .
$$

In $p d$ elastic scattering, an alternative decomposition of the full resolvent is found that is conceptually more revealing. Instead of correlating the plane-wave channel state $\left|\phi_{\alpha}(\mathbf{q}) v_{\alpha}\right\rangle$ in a single step to the full scattering state by $G^{(R)}(Z)$, it may be correlated first to a screened Coulomb state of proton and deuteron by the screened Coulomb potential $W_{\alpha R}^{\text {c.m. between }}$ a proton and the center of mass (c.m.) of the remaining 
neutron-proton ( $n p$ ) pair in channel $\alpha$ through

$$
\begin{aligned}
G_{\alpha R}(Z) & =\left(Z-H_{0}-v_{\alpha}-w_{\alpha R}-W_{\alpha R}^{\text {c.m. }}\right)^{-1}, \\
G_{\alpha R}(Z) & =G_{\alpha}^{(R)}(Z)+G_{\alpha}^{(R)}(Z) T_{\alpha R}^{\text {c.m. }}(Z) G_{\alpha}^{(R)}(Z), \\
T_{\alpha R}^{\text {c.m. }}(Z) & =W_{\alpha R}^{\text {c.m. }}+W_{\alpha R}^{\text {c.m. }} G_{\alpha}^{(R)}(Z) T_{\alpha R}^{\text {c.m. }}(Z),
\end{aligned}
$$

where, in each channel $\alpha$, the potentials $w_{\alpha R}$ and $W_{\alpha R}^{\text {c.m. }}$ are never simultaneously present: When $\alpha$ corresponds to a $p p$ pair, $w_{\alpha R}$ is present and $W_{\alpha R}^{\text {c.m. }}=0$; when $\alpha$ denotes a $n p$ pair, $w_{\alpha R}=0$ and $W_{\alpha R}^{\text {c.m. }}$ is present. The same Coulomb correlation is done explicitly in both initial and final states. Thus the full resolvent can be decomposed, as an alternative to Eq. (4a), as

$$
G^{(R)}(Z)=\delta_{\beta \alpha} G_{\alpha R}(Z)+G_{\beta R}(Z) \tilde{U}_{\beta \alpha}^{(R)}(Z) G_{\alpha R}(Z),
$$

yielding a new form for the full multichannel transition matrix:

$$
\begin{aligned}
U_{\beta \alpha}^{(R)}(Z)= & \delta_{\beta \alpha} T_{\alpha R}^{\text {c.m. }}(Z)+\left[1+T_{\beta R}^{\text {c.m. }}(Z) G_{\beta}^{(R)}(Z)\right] \\
& \times \tilde{U}_{\beta \alpha}^{(R)}(Z)\left[1+G_{\alpha}^{(R)}(Z) T_{\alpha R}^{\text {c.m. }}(Z)\right] .
\end{aligned}
$$

The reduced operator $\tilde{U}_{\beta \alpha}^{(R)}(Z)$ may be calculated through the integral equation

$$
\begin{aligned}
\tilde{U}_{\beta \alpha}^{(R)}(Z)= & \bar{\delta}_{\beta \alpha}\left[G_{\alpha R}^{-1}(Z)+v_{\alpha}\right]+\delta_{\beta \alpha} \mathcal{W}_{\alpha R} \\
& +\sum_{\sigma}\left(\bar{\delta}_{\beta \sigma} v_{\sigma}+\delta_{\beta \sigma} \mathcal{W}_{\beta R}\right) G_{\sigma R}(Z) \tilde{U}_{\sigma \alpha}^{(R)}(Z),
\end{aligned}
$$

which is driven by the strong potential $v_{\alpha}$ and the potential of a three-nucleon nature $\mathcal{W}_{\alpha R}=\sum_{\sigma}\left(\bar{\delta}_{\alpha \sigma} w_{\sigma R}-\delta_{\alpha \sigma} W_{\sigma R}^{\text {c.m. }}\right)$. This potential $\mathcal{W}_{\alpha R}$ accounts for the difference between the direct $p p$ Coulomb interaction and the one that takes place between the proton and the c.m. of the remaining bound as well as unbound $n p$ pair. When calculated between on-shell screened $p d$ Coulomb states, $\tilde{U}_{\beta \alpha}^{(R)}(Z)$ is of short range, even in the infinite $R$ limit.

In the same spirit, the final breakup state to be analyzed may not be reached in a single step; instead it may be correlated first to a screened Coulomb state between the charged particles whose corresponding Coulomb resolvent keeps only the screened Coulomb interaction:

$$
G_{R}(Z)=\left(Z-H_{0}-\sum_{\sigma} w_{\sigma R}\right)^{-1}
$$

In the system of two protons and one neutron, only the channel $\sigma=\rho$, corresponding to a correlated $p p$ pair, contributes to $G_{R}(Z)$,

$$
\begin{aligned}
G_{R}(Z) & =G_{0}(Z)+G_{0}(Z) T_{\rho R}(Z) G_{0}(Z), \\
T_{\rho R}(Z) & =w_{\rho R}+w_{\rho R} G_{0}(Z) T_{\rho R}(Z),
\end{aligned}
$$

making channel $\rho$ the most convenient choice for the description of the final breakup state. Thus, for the purpose of $p d$ breakup, a decomposition of the full resolvent, as an alternative to Eq. (4b), is

$$
\begin{aligned}
G^{(R)}(Z)= & G_{R}(Z) \tilde{U}_{0 \alpha}^{(R)}(Z) G_{\alpha R}(Z) \\
G^{(R)}(Z)= & G_{0}(Z)\left[1+T_{\rho R}(Z) G_{0}(Z)\right] \tilde{U}_{0 \alpha}^{(R)}(Z) \\
& \times\left[1+G_{\alpha}^{(R)}(Z) T_{\alpha R}^{\mathrm{c} . \mathrm{m} .}(Z)\right] G_{\alpha}^{(R)}(Z),
\end{aligned}
$$

where the full breakup transition matrix may be written as

$$
\begin{aligned}
U_{0 \alpha}^{(R)}(Z)= & {\left[1+T_{\rho R}(Z) G_{0}(Z)\right] \tilde{U}_{0 \alpha}^{(R)}(Z) } \\
& \times\left[1+G_{\alpha}^{(R)}(Z) T_{\alpha R}^{\mathrm{c} . \mathrm{m} .}(Z)\right] .
\end{aligned}
$$

The reduced operator $\tilde{U}_{0 \alpha}^{(R)}(Z)$ may be calculated through quadrature,

$$
\tilde{U}_{0 \alpha}^{(R)}(Z)=G_{\alpha R}^{-1}(Z)+v_{\alpha}+\sum_{\sigma} v_{\sigma} G_{\sigma R}(Z) \tilde{U}_{\sigma \alpha}^{(R)}(Z),
$$

from the correspondingly reduced operator $\tilde{U}_{\beta \alpha}^{(R)}(Z)$ of elastic scattering. In the form of Eq. (11a) for the full breakup transition matrix, the external distortions that are due to screened Coulomb in the initial and final states are made explicit. On-shell the reduced operator $\tilde{U}_{0 \alpha}^{(R)}(Z)$ calculated between screened Coulomb distorted initial and final states is of finite range and has two contributions with slightly different range properties:

(i) The contribution $G_{\alpha R}^{-1}(Z)+v_{\alpha}$, when calculated on-shell between initial $p d$ and final three-nucleon states, becomes the three-nucleon potential $\mathcal{W}_{\alpha R}$ and is the longestrange part of breakup, since the $n p$ pair is correlated by the hadronic interaction only in the initial $p d$ state. The corresponding contribution in Ref. [6] is called the pure Coulomb breakup term.

(ii) The remaining part, $\sum_{\sigma} v_{\sigma} G_{\sigma R}(Z) \tilde{U}_{\sigma \alpha}^{(R)}(Z)$, is of shorter range, comparable with the one of the reduced operator $\tilde{U}_{\beta \alpha}^{(R)}(Z)$ for elastic $p d$ scattering.

In the full breakup operator $U_{0 \alpha}^{(R)}(Z)$ the external distortions show up in screened Coulomb waves generated by $[1+$ $\left.G_{\alpha}^{(R)}(Z) T_{\alpha R}^{\mathrm{c} . \mathrm{m} .}(Z)\right]$ in the initial state and by $\left[1+T_{\rho R}(Z) G_{0}(Z)\right]$ in the final state; both wave functions do not have proper limits as $R \rightarrow \infty$. Therefore $U_{0 \alpha}^{(R)}(Z)$ has to be renormalized as the corresponding amplitude for $p d$ elastic scattering $[5,11]$, in order to obtain the results appropriate for the unscreened Coulomb limit. According to Refs. [6,11], the full breakup transition amplitude for initial and final states $\left|\phi_{\alpha}\left(\mathbf{q}_{i}\right) v_{\alpha_{i}}\right\rangle$ and $\left|\phi_{0}\left(\mathbf{p}_{f} \mathbf{q}_{f}\right) v_{0_{f}}\right\rangle, E_{\alpha}\left(q_{i}\right)=E_{0}\left(p_{f} q_{f}\right)$, referring to the strong potential $v_{\alpha}$ and the unscreened Coulomb potential $w_{\alpha}$, is obtained by means of the renormalization of the on-shell breakup transition matrix $U_{0 \alpha}^{(R)}\left(E_{\alpha}\left(q_{i}\right)+i 0\right)$ in the infinite $R$ limit:

$$
\begin{aligned}
& \left\langle\phi_{0}\left(\mathbf{p}_{f} \mathbf{q}_{f}\right) v_{0_{f}}\left|U_{0 \alpha}\right| \phi_{\alpha}\left(\mathbf{q}_{i}\right) v_{\alpha_{i}}\right\rangle \\
& =\lim _{R \rightarrow \infty}\left\{z_{R}^{-\frac{1}{2}}\left(p_{f}\right)\left\langle\phi_{0}\left(\mathbf{p}_{f} \mathbf{q}_{f}\right) v_{0_{f}}\right|\right. \\
& \left.\quad \times U_{0 \alpha}^{(R)}\left(E_{\alpha}\left(q_{i}\right)+i 0\right)\left|\phi_{\alpha}\left(\mathbf{q}_{i}\right) v_{\alpha_{i}}\right\rangle \mathcal{Z}_{R}^{-\frac{1}{2}}\left(q_{i}\right)\right\},
\end{aligned}
$$

where $\mathcal{Z}_{R}\left(q_{i}\right)$ and $z_{R}\left(p_{f}\right)$ are the $p d$ and $p p$ renormalization factors defined below.

As in Ref. [5], here we choose an isospin description for the three baryons in which the nucleons are considered identical. The two-baryon transition matrix $T_{\alpha}^{(R)}(Z)$ becomes an operator, coupling total isospin $\mathcal{T}=\frac{1}{2}$ and $\mathcal{T}=\frac{3}{2}$ states as described in detail in Ref. [5]. Instead of the breakup amplitude given by Eq. (12) we have to use the properly symmetrized 
form,

$$
\begin{aligned}
& \left\langle\phi_{0}\left(\mathbf{p}_{f} \mathbf{q}_{f}\right) v_{0_{f}}\left|U_{0}\right| \phi_{\alpha}\left(\mathbf{q}_{i}\right) v_{\alpha_{i}}\right\rangle \\
& =\sum_{\sigma}\left\langle\phi_{0}\left(\mathbf{p}_{f} \mathbf{q}_{f}\right) v_{0_{f}}\left|U_{0 \sigma}\right| \phi_{\sigma}\left(\mathbf{q}_{i}\right) v_{\sigma_{i}}\right\rangle, \\
& \left\langle\phi_{0}\left(\mathbf{p}_{f} \mathbf{q}_{f}\right) v_{0_{f}}\left|U_{0}\right| \phi_{\alpha}\left(\mathbf{q}_{i}\right) v_{\alpha_{i}}\right\rangle \\
& =\lim _{R \rightarrow \infty}\left\{z_{R}^{-\frac{1}{2}}\left(p_{f}\right)\left\langle\phi_{0}\left(\mathbf{p}_{f} \mathbf{q}_{f}\right) v_{0_{f}}\right|\right. \\
& \left.\left.\times U_{0}^{(R)}\left(E_{\alpha}\left(q_{i}\right)+i 0\right) \mid \phi_{\alpha}\left(\mathbf{q}_{i}\right) v_{\alpha_{i}}\right) \mathcal{Z}_{R}^{-\frac{1}{2}}\left(q_{i}\right)\right\}
\end{aligned}
$$

with $U_{0}^{(R)}(Z)=U_{0 \alpha}^{(R)}(Z)+U_{0 \beta}^{(R)}(Z) P_{231}+U_{0 \gamma}^{(R)}(Z) P_{312}$ for the calculation of observables, $(\alpha \beta \gamma)$ being cyclic and $P_{231}$ and $P_{312}$ being the two cyclic permutations of $(\alpha \beta \gamma)$. The symmetrized breakup transition matrix $U_{0}^{(R)}(Z)$ follows by quadrature,

$$
\begin{aligned}
U_{0}^{(R)}(Z)= & (1+P) G_{0}^{-1}(Z) \\
& +(1+P) T_{\alpha}^{(R)}(Z) G_{0}(Z) U^{(R)}(Z),
\end{aligned}
$$

from the symmetrized multichannel transition matrix $U^{(R)}$ $(Z)=U_{\alpha \alpha}^{(R)}(Z)+U_{\alpha \beta}^{(R)}(Z) P_{231}+U_{\alpha \gamma}^{(R)}(Z) P_{312}$ of elastic $p d$ scattering, satisfying the standard symmetrized form of AGS integral equation (5a), i.e.,

$$
U^{(R)}(Z)=P G_{0}^{-1}(Z)+P T_{\alpha}^{(R)}(Z) G_{0}(Z) U^{(R)}(Z),
$$

with $P=P_{231}+P_{312}$.

The renormalization factors $\mathcal{Z}_{R}\left(q_{i}\right)$ and $z_{R}\left(p_{f}\right)$ in the initial and final channels are diverging phase factors defined in Ref. [10] for a general screening and calculated in Refs. [5,17] for the screened Coulomb potential of Eq. (1), i.e.,

$$
\begin{aligned}
\mathcal{Z}_{R}\left(q_{i}\right) & =e^{-2 i \kappa\left(q_{i}\right)\left[\ln \left(2 q_{i} R\right)-C / n\right]}, \\
z_{R}\left(p_{f}\right) & =e^{-2 i \kappa\left(p_{f}\right)\left[\ln \left(2 p_{f} R\right)-C / n\right]},
\end{aligned}
$$

$\kappa\left(q_{i}\right)=\alpha_{e} M / q_{i}$ and $\kappa\left(p_{f}\right)=\alpha_{e} \mu / p_{f}$ being the $p d$ and $p p$ Coulomb parameters, $M$ and $\mu$ the reduced $p d$ and $p p$ masses, $C \approx 0.5772156649$ the Euler number, and $n$ the exponent in Eq. (1). In $p d$ elastic scattering, the renormalization factors were used in a partial-wave-dependent form that yielded a slight advantage in convergence with $R$ compared with the partial-wave-independent form of Eq. (15). In breakup, the operator $T_{\alpha}^{(R)}(Z) G_{0}(Z) U^{(R)}(Z)$ in Eq. (14a) is calculated on a partial-wave basis, but the on-shell elements of the full breakup operator $U_{0}^{(R)}(Z)$ are calculated on a plane-wave basis. Therefore the renormalization is applicable only in the partial-wave-independent form of Eqs. (15).

The limit in Eq. (13b) has to be performed numerically, but, because of the finite-range nature of the breakup operator, the infinite $R$ limit is reached with sufficient accuracy at rather modest screening radii $R$. Furthermore, the longer-range pure Coulomb breakup part that after symmetrization reads $[1+$ $\left.T_{\rho R}(Z) G_{0}(Z)\right] P v_{\alpha}\left[1+G_{\alpha}^{(R)}(Z) T_{\alpha R}^{\mathrm{c} . \mathrm{m} .}(Z)\right]$ and the remaining shorter-range part can be renormalized with different screening radii, since the limit in Eq. (13b) exist for them separately. The limit for the pure Coulomb breakup part can even be carried out explicitly, since the renormalization of the screened Coulomb waves yields the corresponding unscreened Coulomb waves accessible in configuration space; thus the integral can be carried out numerically in configuration space as was done indeed in Ref. [6]. However, we find such a procedure unnecessary when our standard screening function is used. In fact, in most cases there is even no necessity for splitting the full breakup amplitude into pure Coulomb and Coulomb-modified short-range parts, the only exception being the kinematical situations characterized by small momentum transfer in the $p p$ subsystem that are sensitive to the Coulomb interaction at larger distances.

The practical implementation of the outlined calculational scheme faces a technical difficulty. We solve Eq. (14b) on a partial-wave basis. The partial-wave expansion of the screened Coulomb potential converges rather slowly. In this context, the perturbation theory for higher two-baryon partial waves developed in Ref. [18] is a very efficient and reliable technical tool for treating the screened Coulomb interaction in high partial waves. We vary the dividing line between partial waves included exactly and perturbatively in order to test the convergence and thereby establish the validity of the procedure. Furthermore, the partial-wave convergence becomes slightly faster when lowest-order screened Coulomb contributions in $U_{0}^{(R)}(Z)$ are replaced with the respective plane-wave results, i.e.,

$$
U_{0}^{(R)}(Z)=\left[U_{0}^{(R)}(Z)-(1+P) w_{\alpha R} P\right]+(1+P) w_{\alpha R} P,
$$

where the first term converges with respect to partial waves faster than $U_{0}^{(R)}(Z)$ itself and the second term is calculated without partial-wave decomposition.

With respect to the partial-wave expansion in the actual calculations of this paper, we obtain fully converged results by taking into account the screened Coulomb interaction in two-baryon partial waves with pair orbital angular momentum $L \leqslant 15$; orbital angular momenta $9 \leqslant L \leqslant 15$ can safely be treated perturbatively. The above values refer to the screening radius $R=30 \mathrm{fm}$; for smaller screening radii the convergence in orbital angular momentum is faster. The hadronic interaction is taken into account in two-baryon partial waves with total angular momentum $I \leqslant 5$. Both three-baryon total isospin $\mathcal{T}=1 / 2$ and $\mathcal{T}=3 / 2$ states are included. The maximal three-baryon total angular momentum $\mathcal{J}$ considered is $61 / 2$. Figures 1-3 study the convergence of our method with increasing screening radius $R$ according to Eq. (13b). All the calculations of this section are based on CD Bonn as the hadronic interaction. The kinematical final-state configurations are characterized in a standard way by the polar angles of the two protons and by the azimuthal angle between them $\left(\theta_{1}, \theta_{2}, \varphi_{2}-\varphi_{1}\right)$. We show several characteristic examples referring to $p d$ breakup at $13-\mathrm{MeV}$ proton lab energy and at $130-\mathrm{MeV}$ deuteron lab energy. The convergence is impressive for the spin-averaged differential cross section as well as for the spin observables in most kinematical situations, as demonstrated in Figs. 1 and 2. The screening radius $R=20 \mathrm{fm}$ is sufficient; it is only in the top plot of Fig. 1 that the curves for $R=20 \mathrm{fm}$ and $R=30 \mathrm{fm}$ are graphically distinguishable. The exception requiring larger screening radii is the differential cross section in kinematical situations characterized by very low $p p$ relative energy $E_{p p}$, i.e., close to the $p p$ final-state 

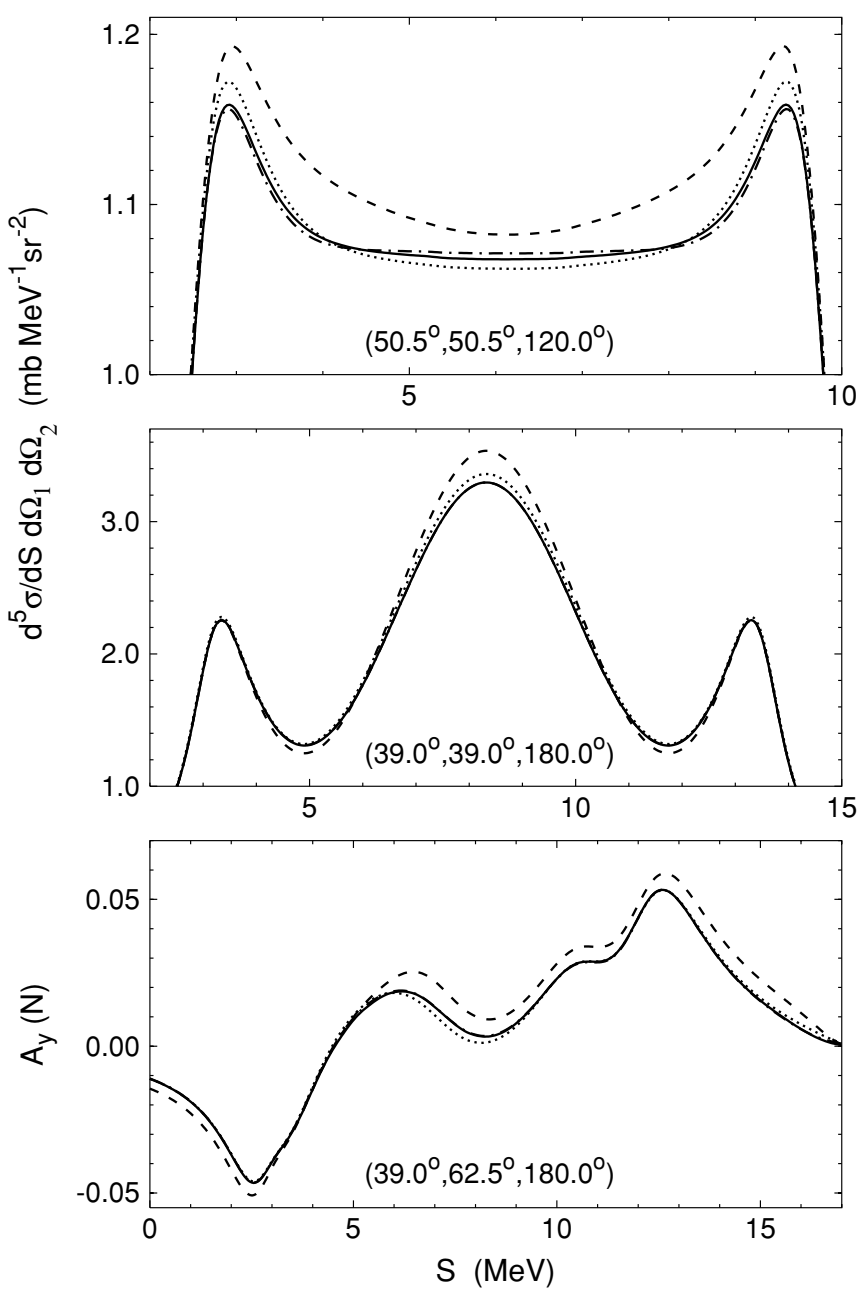

FIG. 1. Convergence of the $p d$ breakup observables with screening radius $R$. The differential cross section and the proton analyzing power $A_{y}(N)$ for $p d$ breakup at $13-\mathrm{MeV}$ proton lab energy are shown as functions of the arc length $S$ along the kinematical curve. Results for the CD Bonn potential obtained with screening radius $R=10 \mathrm{fm}$ (dotted curves), $20 \mathrm{fm}$ (dash-dotted curves), and $30 \mathrm{fm}$ (solid curves) are compared. Results without Coulomb (dashed curves) are given as reference for the size of the Coulomb effect.

interaction ( $p p$-FSI) regime, as shown in Fig. 3. In there, the $p p$ repulsion is responsible for decreasing the cross section, converting the $p p$-FSI peak obtained in the absence of Coulomb into a minimum with zero cross section at $p_{f}=0$, i.e., for $E_{p p}=0$. A similar convergence problem also takes place in $p p$ scattering at very low energies, as discussed in Ref. [5]. In fact, screening and renormalization procedures cannot be applied at $p_{f}=0$, since the renormalization factor $z_{R}\left(p_{f}=0\right)$ is ill defined. Therefore an extrapolation has to be used to calculate the observables at $p_{f}=0$, which works pretty well since the observables vary smoothly with $p_{f}$. In Fig. 3 the fully converged result would start at zero for $E_{p p}=0$.

The seen Coulomb effects and their physics implications are discussed in Sec. III.

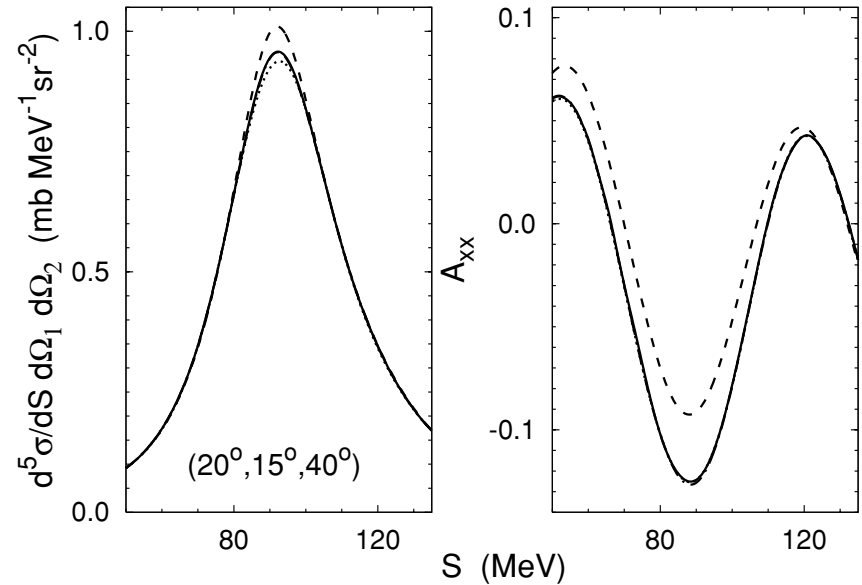

FIG. 2. Convergence of the $p d$ breakup observables with screening radius $R$. The differential cross section and the deuteron analyzing power $A_{x x}$ for $p d$ breakup at $130 \mathrm{MeV}$ are shown. Curves as in Fig. 1.

\section{B. Three-body e.m. disintegration of ${ }^{3} \mathrm{He}$}

For the description of the considered e.m. processes, the matrix element $\left\langle\psi_{0}^{(-)}\left(\mathbf{p}_{f} \mathbf{q}_{f}\right) v_{0_{f}}\left|j^{\mu}\left(\mathbf{Q}, \mathbf{K}_{+}\right)\right| B\right\rangle$ of the e.m. current operator between the three-nucleon bound state and the breakup scattering state has to be calculated. The calculation of that matrix element without Coulomb and the meaning of the momenta $\mathbf{Q}$ and $\mathbf{K}_{+}$are discussed in great length in Refs. $[19,20]$. This subsection discusses only the modification that arises because of the inclusion of the Coulomb interaction between the charged baryons. Coulomb is included as a screened potential, and the dependence of the bound and

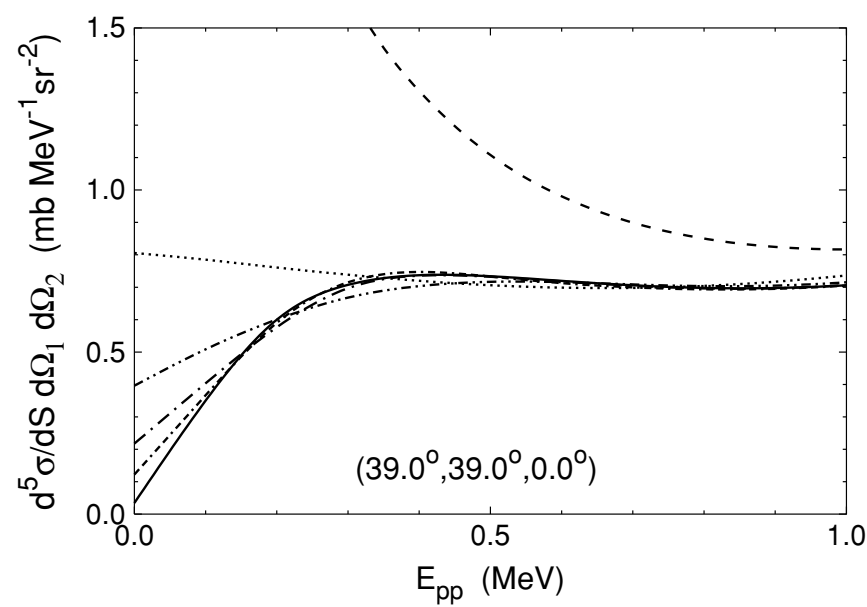

FIG. 3. Convergence of the $p d$ breakup observables with screening radius $R$. The differential cross section for $p d$ breakup at 13-MeV proton lab energy in the $p p$-FSI configuration is shown as function of the relative $p p$ energy $E_{p p}$. Results obtained with screening radius $R=10 \mathrm{fm}$ (dotted curves), $20 \mathrm{fm}$ (dashed-double-dotted curves), $30 \mathrm{fm}$ (dashed-dotted curves), $40 \mathrm{fm}$ (double-dashed-dotted curves), and $60 \mathrm{fm}$ (solid curves) are compared. Results without Coulomb (dashed curves) are given as reference for the size of the Coulomb effect. 


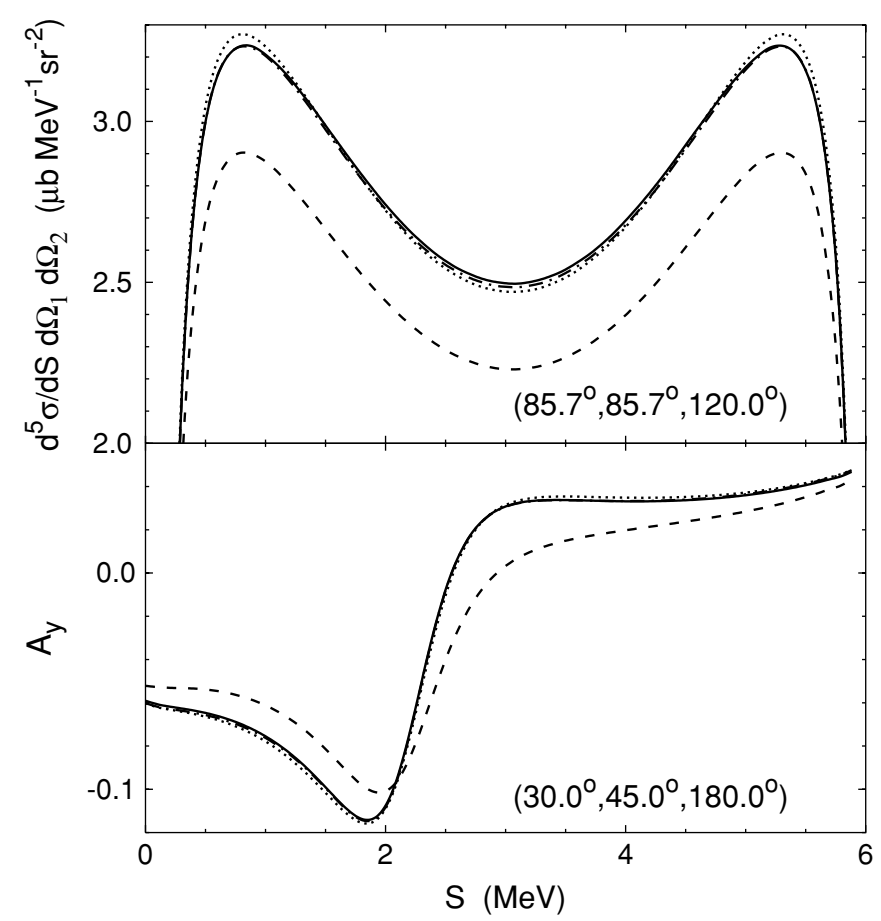

FIG. 4. Convergence of the ${ }^{3} \mathrm{He}(\gamma, p p) n$ reaction observables with screening radius $R$. The differential cross section and the target analyzing power $A_{y}$ at $15-\mathrm{MeV}$ photon lab energy are shown. Curves as in Fig. 1.

scattering states, i.e., $\left|B^{(R)}\right\rangle$ and $\left|\psi_{0}^{( \pm)(R)}\left(\mathbf{p}_{f} \mathbf{q}_{f}\right) v_{0_{f}}\right\rangle$, on the screening radius $R$ is notationally made explicit. In analogy to $p d$ breakup, the current matrix element referring to the unscreened Coulomb potential is obtained by means of renormalization of the matrix element referring to the screened Coulomb potential in the infinite $R$ limit:

$$
\begin{aligned}
& \left\langle\psi_{0}^{(-)}\left(\mathbf{p}_{f} \mathbf{q}_{f}\right) v_{0_{f}}\left|j^{\mu}\left(\mathbf{Q}, \mathbf{K}_{+}\right)\right| B\right\rangle \\
& \quad=\lim _{R \rightarrow \infty}\left\{z_{R}^{-\frac{1}{2}}\left(p_{f}\right)\left\langle\psi_{0}^{(-)(R)}\left(\mathbf{p}_{f} \mathbf{q}_{f}\right) v_{0_{f}}\left|j^{\mu}\left(\mathbf{Q}, \mathbf{K}_{+}\right)\right| B^{(R)}\right\rangle\right\} .
\end{aligned}
$$

The renormalization factor $z_{R}\left(p_{f}\right)$ is the same as that used in $p d$ breakup for the final state. Because of the short-range nature of $j^{\mu}\left(\mathbf{Q}, \mathbf{K}_{+}\right)\left|B^{(R)}\right\rangle$, the limit $R \rightarrow \infty$ is reached with sufficient accuracy at finite screening radii $R$. The presence of the bound-state wave function in the matrix element strongly suppresses the contribution of the screened Coulomb interaction in high partial waves, i.e., two-baryon partial waves with orbital angular momentum $L \leqslant 6$ are sufficient for convergence. The other quantum-number-related cutoffs in the partial-wave dependence of the matrix element are the same as in Refs. [19,20], i.e., $I \leqslant 4, \mathcal{J} \leqslant 15 / 2$ for photoreactions, and $I \leqslant 3, \mathcal{J} \leqslant 35 / 2$ for inelastic electron scattering from ${ }^{3} \mathrm{He}$. All calculations include both total isospin $\mathcal{T}=1 / 2$ and $\mathcal{T}=3 / 2$ states.

Figures 4 and 5 study the convergence of our method with increasing screening radius $R$ for the three-body photodisintegration of ${ }^{3} \mathrm{He}$ at $15-$ and $55-\mathrm{MeV}$ photon lab energy. The calculations are again based on CD Bonn as the hadronic

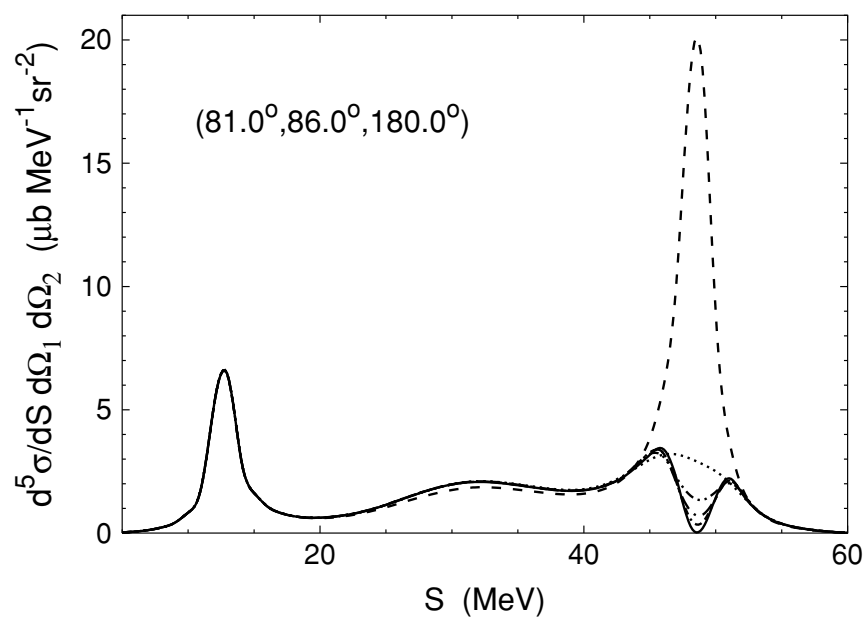

FIG. 5. Convergence of the ${ }^{3} \mathrm{He}(\gamma, p n) p$ reaction observables with screening radius $R$. The differential cross section at $55-\mathrm{MeV}$ photon lab energy in the $p p$-FSI configuration is shown. Curves as in Fig. 3.

interaction and the currents from Refs. [19,20]. We show the differential cross section and the target-analyzing power $A_{y}$ for selected kinematic configurations. The convergence is again extremely good and quite comparable with $p d$ breakup; the screening radius $R=20 \mathrm{fm}$ is fully sufficient in most cases. The only exceptional cases, as in the $p d$ breakup, are $p p$-FSI regimes, as shown in Fig. 5. The convergence with increasing screening radius $R$ is the same for the three-body electrodisintegration of ${ }^{3} \mathrm{He}$; we therefore omit a corresponding figure.

\section{RESULTS}

We base our calculations on the two-baryon coupledchannel potential CD Bonn $+\Delta$ with and without Coulomb and use the CD Bonn potential with Coulomb as a purely nucleonic reference. We use the charge and current operators of Refs. [19,20], which are appropriate for the underlying dynamics. In contrast to Ref. [5], here we do not include one-nucleon relativistic charge corrections for photoreactions, since their effect on the considered observables is very small.

Obviously we have many more predictions than it is possible to show. Therefore we make a selection of the most interesting predictions that illustrate the message we believe the results tell us. Readers are welcome to obtain the results for their favorite data from us.

\section{A. Proton-deuteron breakup}

Figures 6-9 give our results for the fivefold differential cross section at 10.5-, 13-, 19-, and 65-MeV proton lab energies in the standard space star, collinear, quasi-free-scattering (QFS), and $n p$ final-state interaction ( $n p-\mathrm{FSI})$ configurations, for which there are available experimental data. Although the inclusion of Coulomb slightly improves the agreement with 


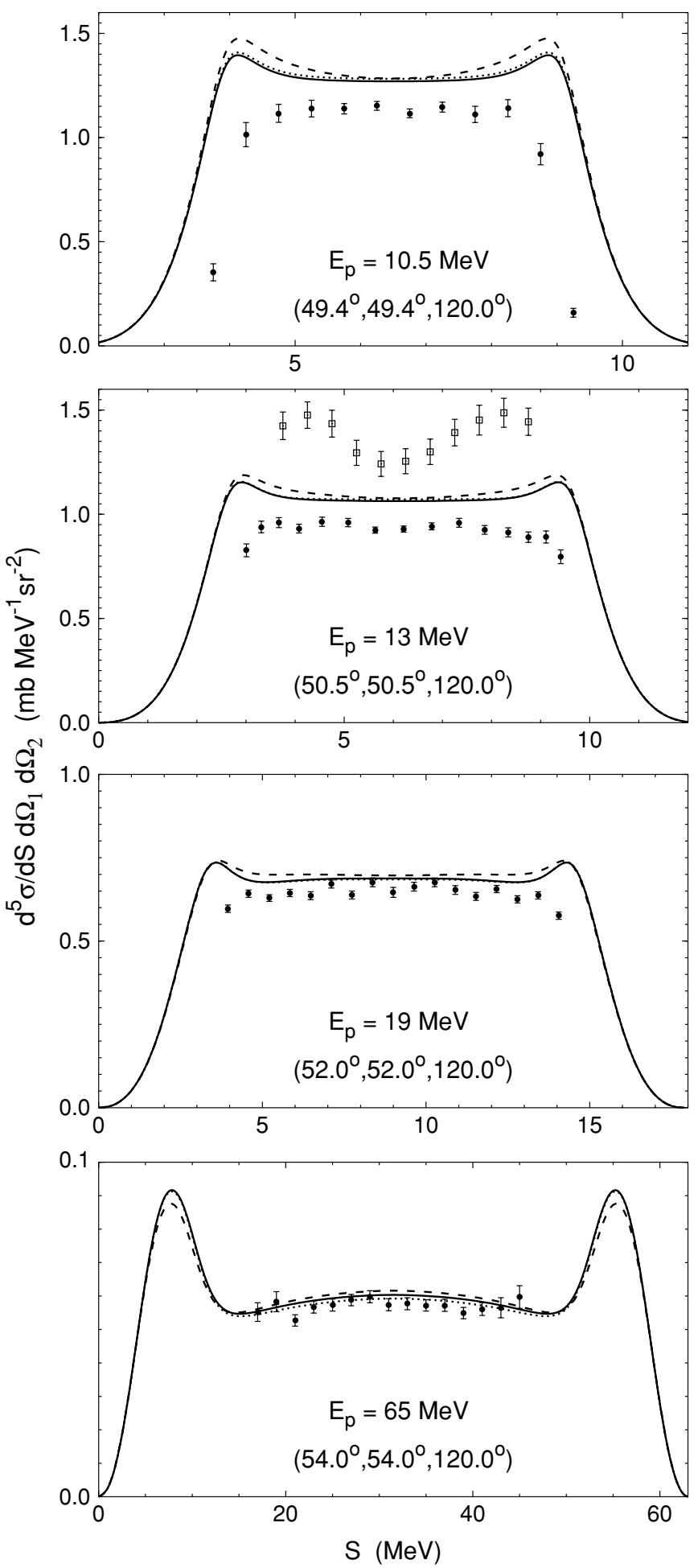

FIG. 6. Differential cross section for space star configurations as a function of the arc length $S$ along the kinematical curve. Results including $\Delta$-isobar excitation and the Coulomb interaction (solid curves) are compared with results without Coulomb (dashed curves). To show the size of the $\Delta$-isobar effect, the purely nucleonic results including Coulomb are also shown (dotted curves). The experimental $p d$ data (circles) are from Ref. [21] at $10.5 \mathrm{MeV}$, from Ref. [22] at $13 \mathrm{MeV}$, from Ref. [23] at $19 \mathrm{MeV}$, from Ref. [24] at $65 \mathrm{MeV}$, and $n d$ data at $13 \mathrm{MeV}$ (squares) from Ref. [25].
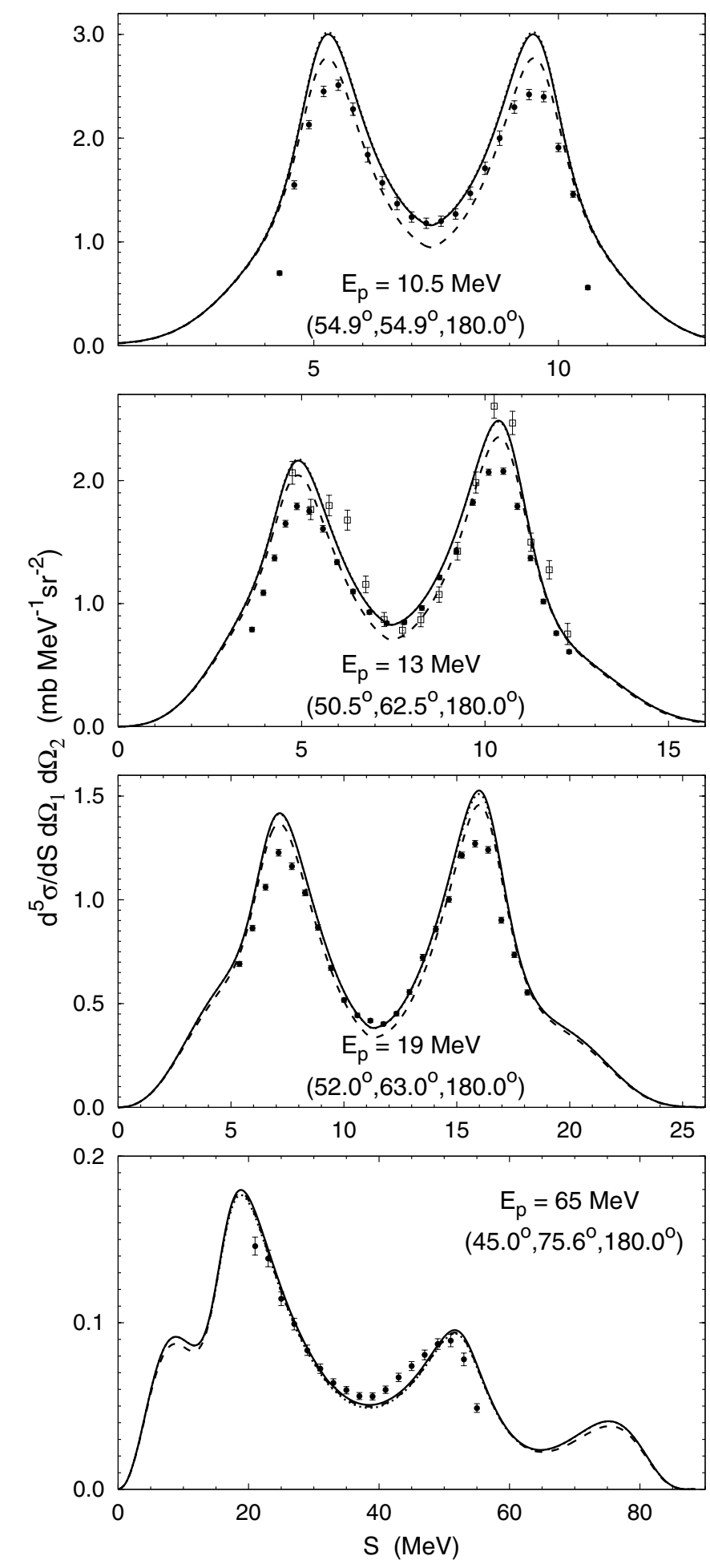

FIG. 7. Differential cross section for collinear configurations. Curves and experimental data as in Fig. 6, except for 65-MeV data from Ref. [26].

data in the space star configurations in Fig. 6, the Coulomb effect is far too small to reproduce the difference between $p d$ and $n d$ data and to resolve the so-called space star anomaly at $13 \mathrm{MeV}$. The inclusion of Coulomb clearly improves the description of the data around the collinear points at lower energies, i.e., at the minima in Fig. 7. The remaining 

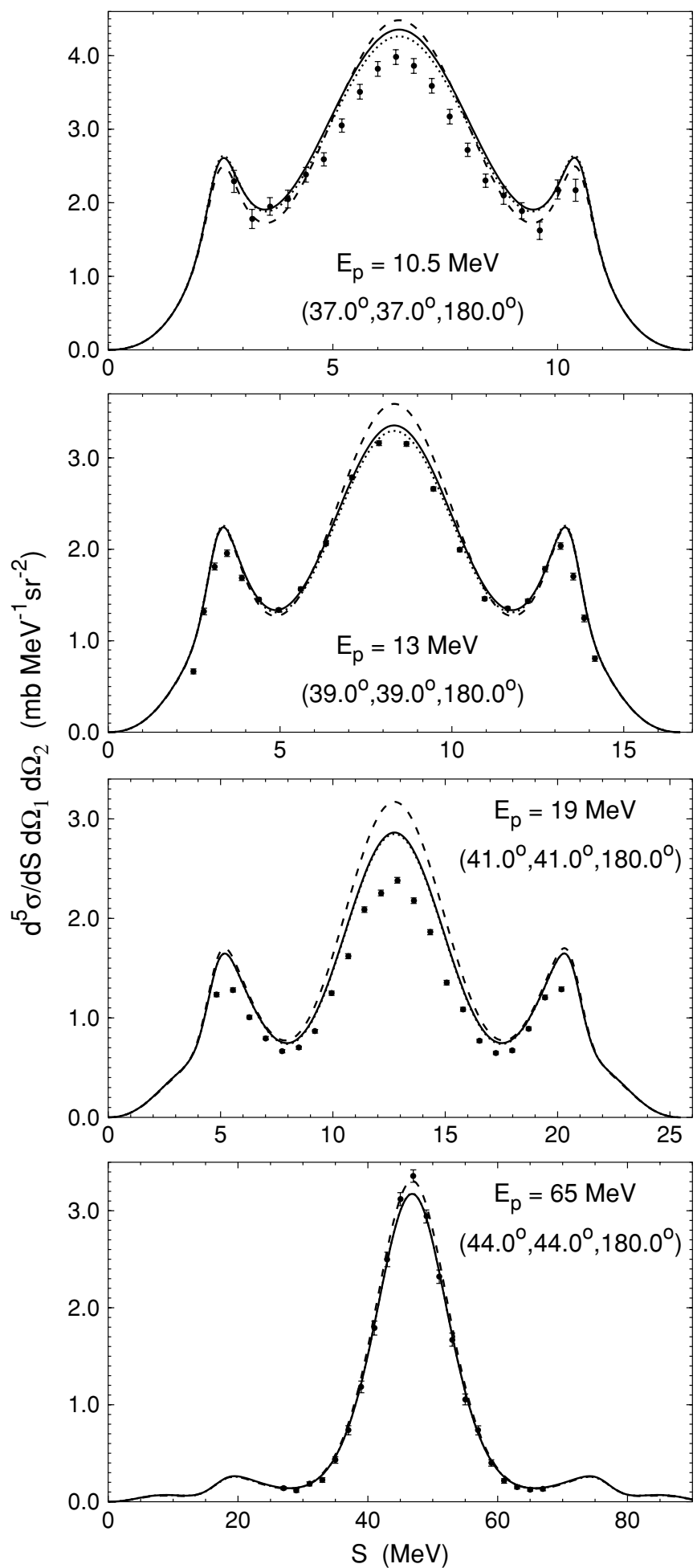

FIG. 8. Differential cross section for QFS configurations. Curves and experimental data as in Fig. 6, except for $65-\mathrm{MeV}$ data from Ref. [27].

discrepancies around the peaks are probably due to the finite geometry, not taken into account in our calculations owing to the lack of information on experimental details, but may also be due to the underlying hadronic interaction. The inclusion of Coulomb decreases the differential cross section around
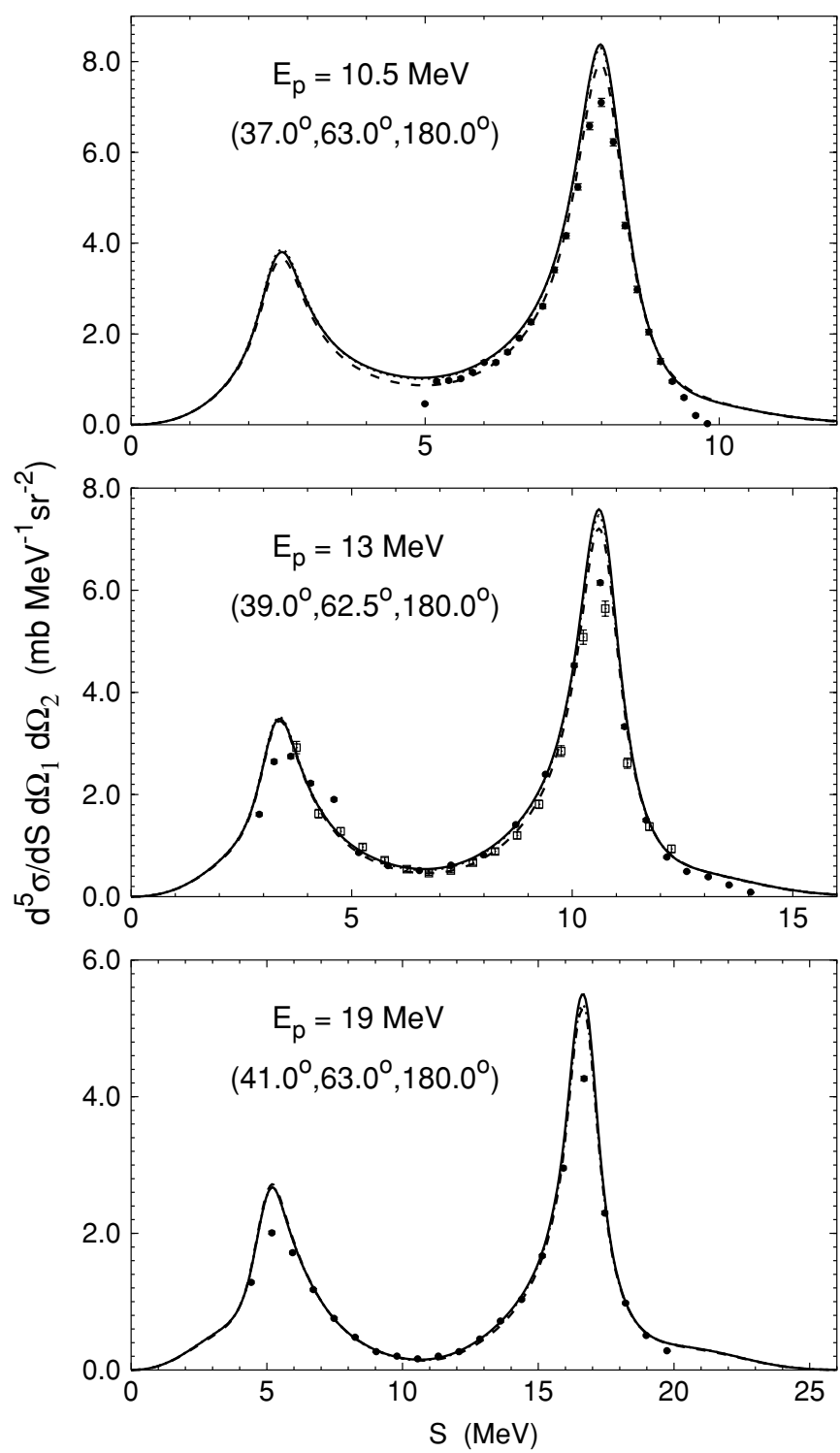

FIG. 9. Differential cross section for $n p$-FSI configurations. Curves and experimental data as in Fig. 6.

the QFS peaks, i.e., around the central peaks in Fig. 8; those changes are supported by the data at lower energies. In the $n p$-FSI configurations of Fig. 9 the Coulomb effect is rather insignificant.

The Coulomb effect on proton-analyzing powers in the considered kinematical configurations is usually small, on the scale of the experimental error bars. We therefore show in Fig. 10 only few collinear configurations.

Recently, $p d$ breakup has been measured at $130-\mathrm{MeV}$ deuteron lab energy in a variety of kinematical configurations [28]. In some of them we find significant Coulomb effects for the differential cross section as well as for the deuteronanalyzing powers. Examples are shown in Figs. 11 and 12. By and large the agreement between theoretical predictions and experimental data is improved. The $p p$-FSI repulsion is responsible for lowering the peak of the differential cross section in the configuration $\left(15^{\circ}, 15^{\circ}, 40^{\circ}\right)$ in Fig. 11, left-hand 

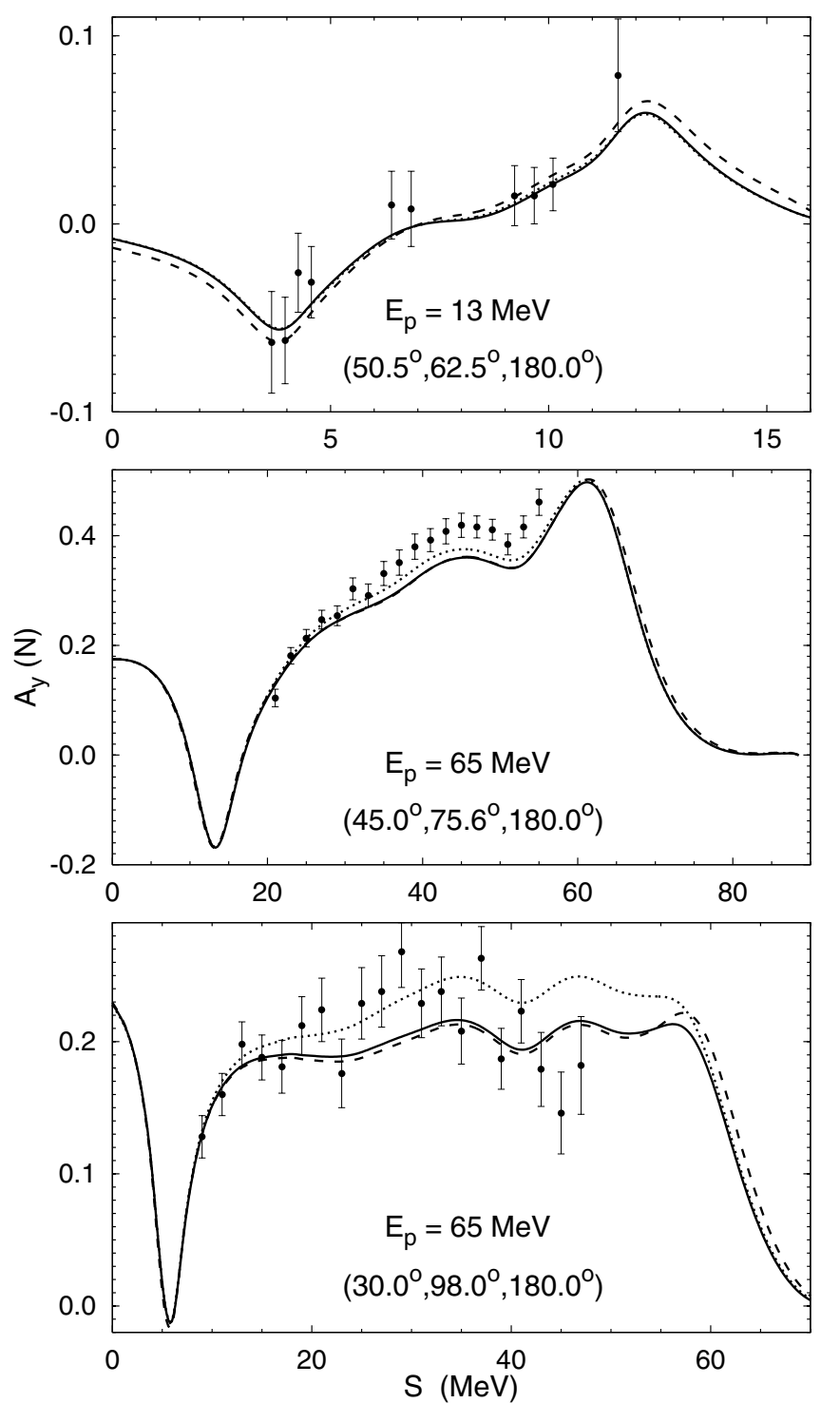

FIG. 10. Proton-analyzing power for collinear configurations at 13- and 65-MeV proton lab energy. Curves and experimental data as in Fig. 7.

panel, where the relative $p p$ energy is rather low at the peak. In contrast, the relative $p p$ energy gets considerably increased as one changes the azimuthal angle to $160^{\circ}$ in Fig. 11, right-hand panel, leading to an increase of the differential cross section that is due to Coulomb. Since the total breakup cross section at this energy, corresponding to $65-\mathrm{MeV}$ proton lab energy, is almost unaffected by Coulomb, as shown in Fig. 13, one may expect in given configurations an increase of the cross section that is due to Coulomb to compensate for the sharp decrease of the cross section in the vicinity of $p p$-FSI points. Figure 12 shows deuteron tensor-analyzing powers $A_{x x}$ and $A_{y y}$ for the same configurations for which experimental data may become available soon [30].

Compared with the results of Ref. [6], based on a simple hadronic $S$-wave potential, we see a rough qualitative agreement in most cases. Quantitatively, the Coulomb effect we observe is smaller than the one of Ref. [6].
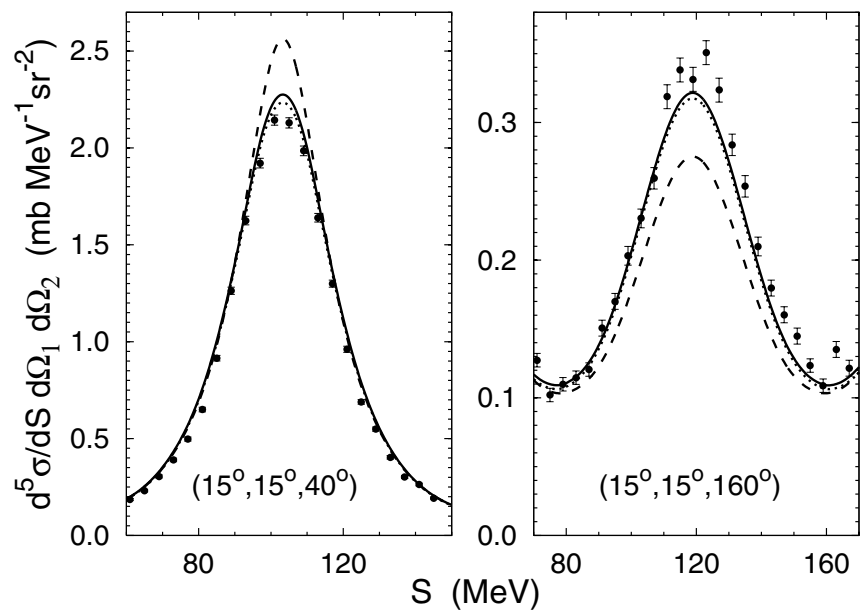

FIG. 11. Differential cross section for $p d$ breakup at $130-\mathrm{MeV}$ deuteron lab energy. Curves as in Fig. 6. The experimental data are from Ref. [28].

Figures 6-13 recall also the $\Delta$-isobar effect on observables, which, in most cases we studied, is much smaller than the Coulomb effect. As expected, the $\Delta$-isobar effect on polarization observables is more significant than on the differential cross sections, which confirms previous findings [13].

\section{B. Three-body e.m. disintegration of ${ }^{3} \mathrm{He}$}

Experimental data for three-body photodisintegration of ${ }^{3} \mathrm{He}$ are very scarce; we therefore show in Fig. 14 only two examples referring to the semi-inclusive ${ }^{3} \mathrm{He}(\gamma, p n) p$ reaction at $55-$ and $85-\mathrm{MeV}$ photon lab energy. The semi-inclusive fourfold differential cross section is obtained from the standard fivefold differential cross section by integrating over the

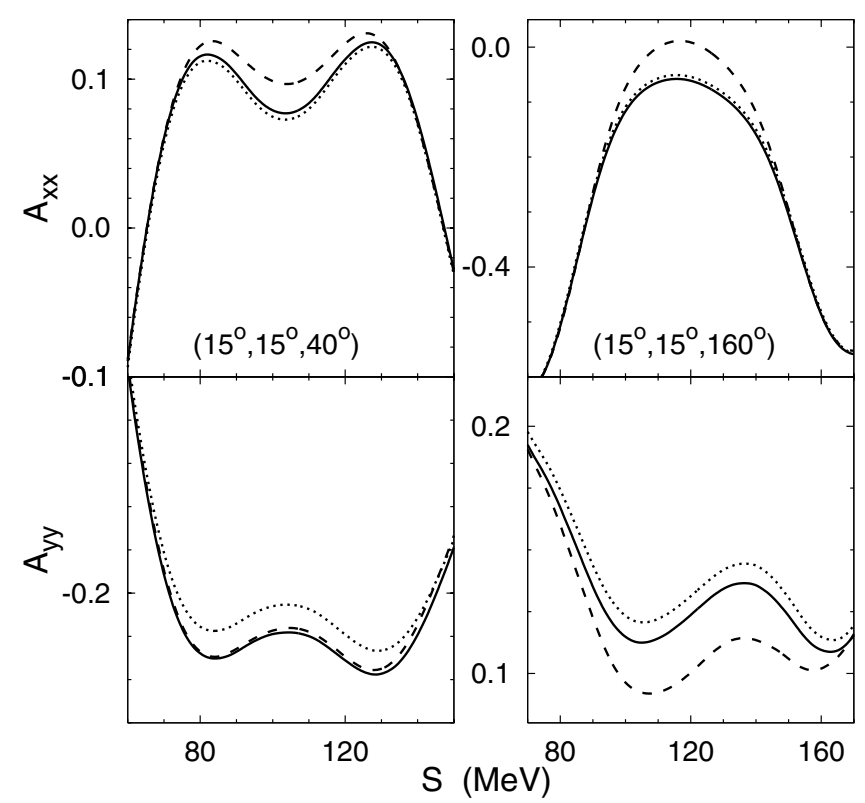

FIG. 12. Deuteron-analyzing powers for $p d$ breakup at $130-\mathrm{MeV}$ deuteron lab energy. Curves as in Fig. 6. 


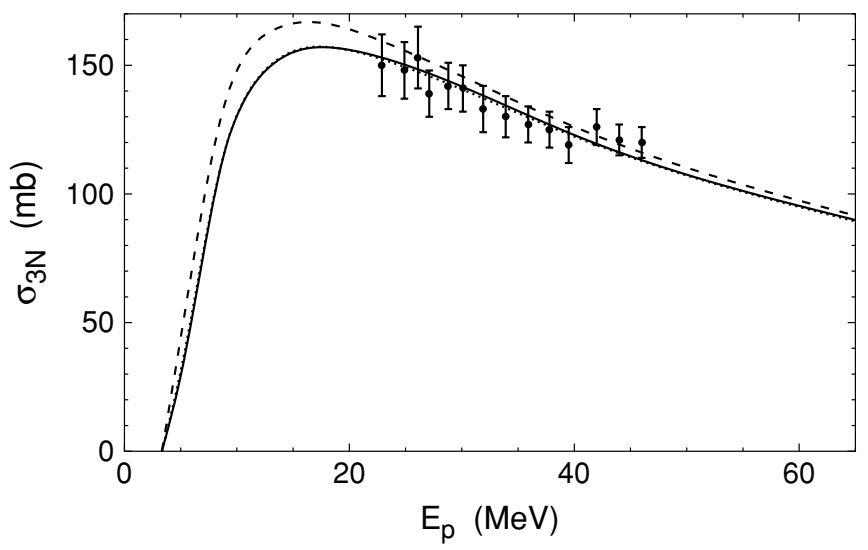

FIG. 13. Total cross section for $p d$ breakup as function of the proton lab energy. Curves as in Fig. 6. The experimental data are from Ref. [29].

kinematical curve $S$. For scattering angles corresponding to the peak of the fourfold differential cross section, the region of the phase space to be integrated over contains a $p p$-FSI regime, in which the $p p$-FSI peak obtained without Coulomb is converted into a minimum as shown in Fig. 5. Therefore the fourfold differential cross section in Fig. 14 is also significantly reduced by the inclusion of Coulomb, clearly improving the agreement with the data. A similar Coulomb effect of the same origin is shown in Fig. 15 for the semi-inclusive threefold differential cross section for the ${ }^{3} \mathrm{He}(\vec{\gamma}, n) p p$ reaction at $15-\mathrm{MeV}$ photon lab energy. In contrast, the photon-analyzing power remains almost unchanged by the inclusion of Coulomb. The experiment measuring this reaction is in progress [32], but the data are not available yet. Again the importance of the $\Delta$-isobar degree of freedom is considerably smaller than the effect of Coulomb.

The available data of three-nucleon electrodisintegration of ${ }^{3} \mathrm{He}$ refer to fully inclusive observables. In Fig. 16 we

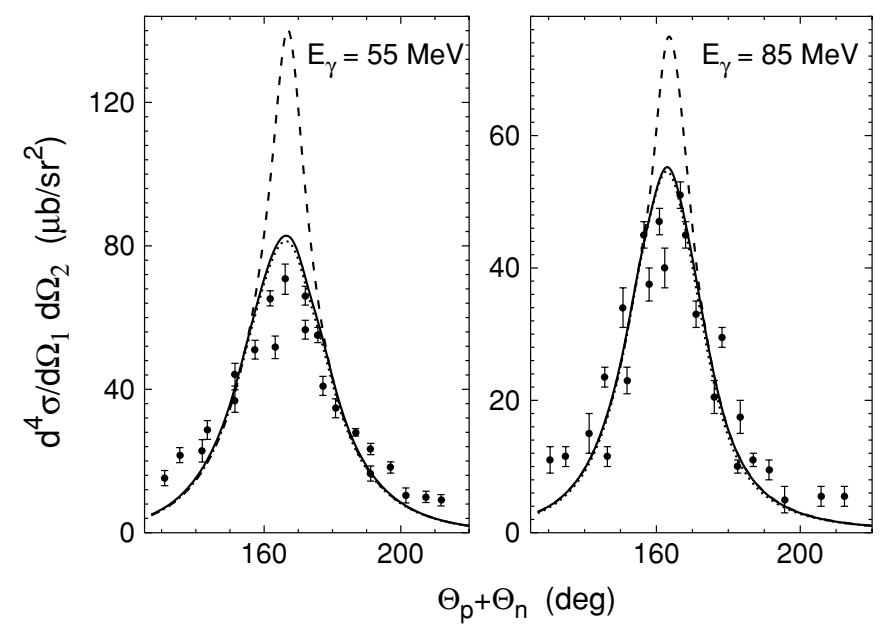

FIG. 14. The semi-inclusive fourfold differential cross section for the ${ }^{3} \mathrm{He}(\gamma, p n) p$ reaction at $55-$ and $85-\mathrm{MeV}$ photon lab energy as a function of the $n p$ opening angle $\theta_{p}+\theta_{n}$ with $\theta_{p}=81^{\circ}$. Curves as in Fig. 6. The experimental data are from Ref. [31].

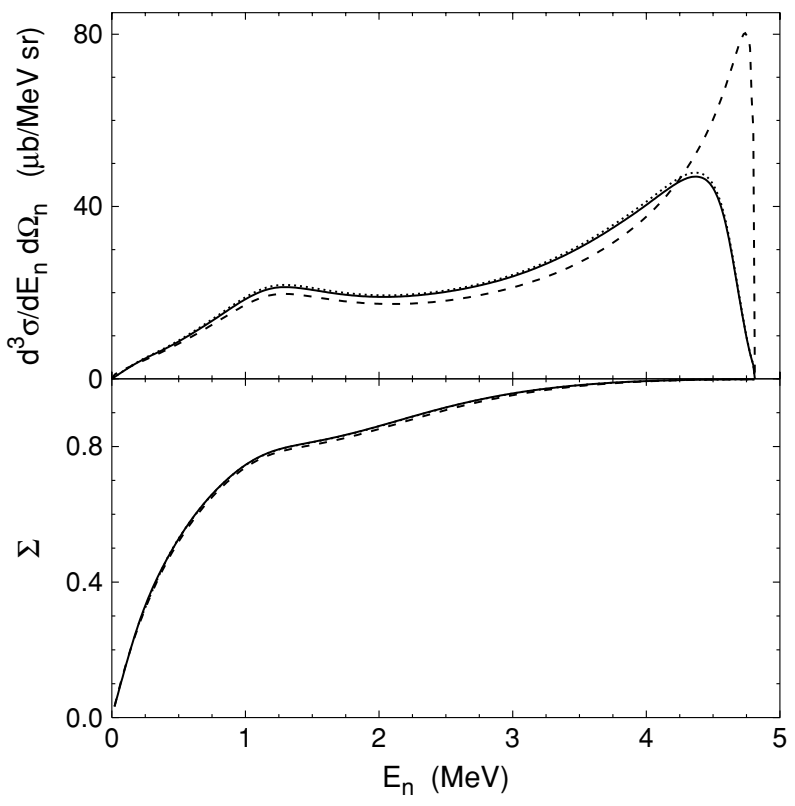

FIG. 15. The semi-inclusive threefold differential cross section and photon-analyzing power $\Sigma$ for the ${ }^{3} \mathrm{He}(\vec{\gamma}, n) p p$ reaction at $15-\mathrm{MeV}$ photon lab energy as a function of the neutron energy $E_{n}$ for the neutron scattering angle $\theta_{n}=90^{\circ}$. Curves as in Fig. 6 .

show ${ }^{3} \mathrm{He}$ inclusive longitudinal and transverse response functions $R_{L}$ and $R_{T}$ as examples. Although the Coulomb effect may be large in particular kinematic regions, it is rather insignificant for the total cross section and therefore also for response functions. Only the transverse response function near

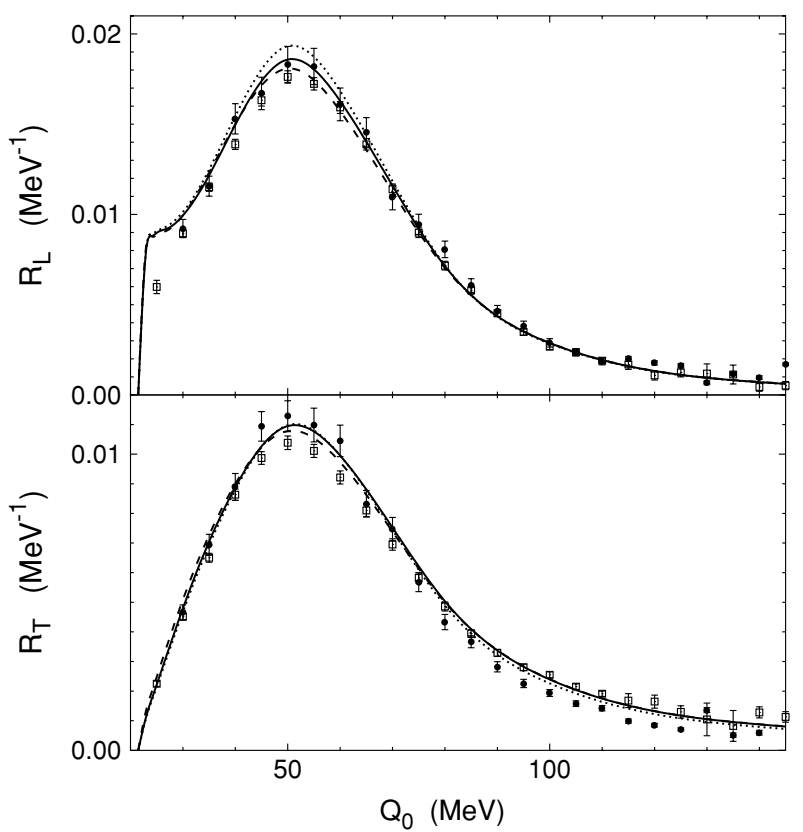

FIG. 16. ${ }^{3} \mathrm{He}$ inclusive longitudinal and transverse response functions $R_{L}$ and $R_{T}$ for the momentum transfer $|\mathbf{Q}|=300 \mathrm{MeV}$ as functions of the energy transfer $Q_{0}$. Curves as in Fig. 6. The experimental data are from Ref. [33] (circles) and from Ref. [34] (squares). 


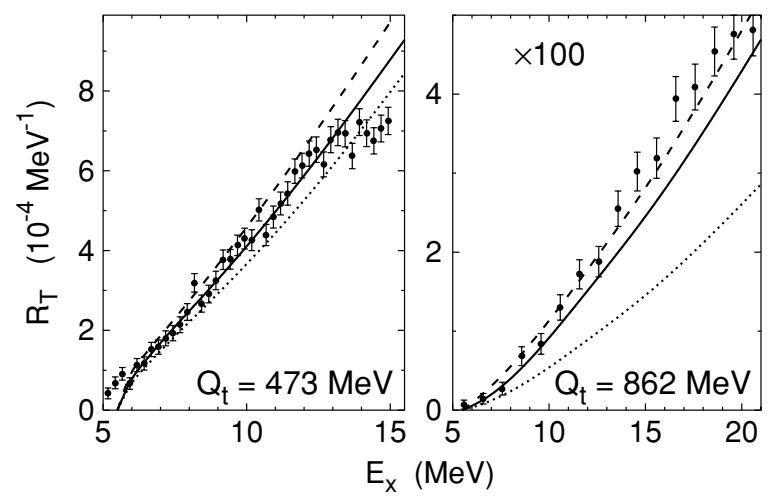

FIG. 17. ${ }^{3} \mathrm{He}$ inclusive transverse response function $R_{T}$ near threshold as a function of the excitation energy $E_{x} ; Q_{t}$ is the value of three-momentum transfer at threshold. Curves as in Fig. 6. The experimental data are from Ref. [35].

threshold is affected more visibly, as shown in Fig. 17; at higher momentum transfer there is also quite a large $\Delta$-isobar effect.

\section{SUMMARY}

In this paper we show how the Coulomb interaction between charged baryons can be included in the momentum-space description of proton-deuteron breakup and of the three-body e.m. disintegration of ${ }^{3} \mathrm{He}$ by using the screening and renormalization approach. The theoretical framework is the AGS integral equation [9]. The calculations are done on the same level of accuracy and sophistication as for the corresponding neutron-deuteron and ${ }^{3} \mathrm{H}$ reactions. The conclusions of the paper refer to the developed technique and to the physics results obtained with that technique.

Technically, the idea of screening and renormalization is that of Refs. [6,10,11]. However, our practical realization differs quite significantly from that of Ref. [6]:

(i) We use modern hadronic interactions, CD Bonn and CD Bonn $+\Delta$, in contrast to the simple $S$-wave separable potentials of Ref. [6]. Our use of the full potential requires the standard form of the three-particle equations, different from the quasiparticle approach of Ref. [6].

(ii) We do not approximate the screened Coulomb transition matrix by the screened Coulomb potential.

(iii) The quasiparticle approach of Ref. [6] treats the screened Coulomb potential between the protons without partialwave expansion and therefore has no problems with the slow convergence of that expansion. Our solution of three-nucleon equations proceeds on the partialwave basis and therefore faces the slow partial-wave convergence of the Coulomb interaction between the charged baryons. However, we are able to obtain fully converged results by choosing a special form of the screening function and by using the perturbation theory of Ref. [18] for treating the screened Coulomb transition matrix in high partial waves. This would not be possible, if we had used Yukawa screening as in Ref. [6], for two reasons: (a) The convergence with respect to screening would require much larger radii $R$ and (b) the larger values of $R$ would necessitate the solution of the AGS equation with much higher angular-momentum states.

(iv) Our method for including the Coulomb interaction is efficient. Although the number of the isospin triplet partial waves to be taken into account is considerably higher than in the case without Coulomb, the required computing time increases by only a factor of $3-4$ for each screening radius $R$, because of the use of perturbation theory for high partial waves.

The obtained results are fully converged with respect to the screening and with respect to the quantum-number cutoffs; they are therefore well checked for their validity. The employed technique becomes cumbersome in kinematical regions with very low relative $p p$ energy, i.e., $p p$ c.m. energies below $0.1 \mathrm{MeV}$, because of the need for quite large screening radii.

As to physics, the Coulomb effect in $p d$ breakup and in the three-body e.m. disintegration of ${ }^{3} \mathrm{He}$ is extremely important in kinematical regimes close to $p p$-FSI. There the $p p$ repulsion converts the $p p$-FSI peak obtained in the absence of Coulomb into a minimum with zero cross section. This significant change of the cross-section behavior has important consequences in nearby configurations where one may observe instead an increase in the cross section that is due to Coulomb. This phenomenon is independent of the beam energy and depends solely on specific momentum distributions of the three-nucleon final state. Therefore, unlike in $p d$ elastic scattering in which the Coulomb contribution decreases with the beam energy until it is confined to the forward direction, in three-body breakup large Coulomb effects may always be found in specific configurations besides $p p$-FSI, even at high beam energies.

Another important consequence of this work is that we can finally ascertain with greater confidence the quality of two- and three-nucleon force models one uses to describe $p d$ observables; any disagreement with high-quality $p d$ data may now be solely attributed to the underlying nuclear interaction. In the framework of the present study we reanalyzed the contribution of $\Delta$-isobar degrees of freedom to three-body breakup observables. The largest $\Delta$ effects take place in analyzing powers for given configurations. Nevertheless, the lack of high-quality analyzing power data on a broad spectrum of configurations prevents a full evaluation of the $\Delta$ effects in $p d$ breakup. The situation is even worse in the threebody photodisintegration of ${ }^{3} \mathrm{He}$, for which there are neither kinematically complete experiments without polarization nor any analyzing power data available.

\section{ACKNOWLEDGMENTS}

The authors thank St. Kistryn and H. Paetz gen Schieck for providing experimental data. A.D. is supported by Fundação para a Ciência e a Techologia (FCT) grant SFRH/BPD/14801/2003, A.C.F. in part by FCT grant POCTI/FNU/37280/2001, and P.U.S. in part by Deutsche Forschungsgemeinschaft grant Sa 247/25. 
[1] E. O. Alt, A. M. Mukhamedzhanov, M. M. Nishonov, and A. I. Sattarov, Phys. Rev. C 65, 064613 (2002).

[2] A. Kievsky, M. Viviani, and S. Rosati, Phys. Rev. C 64, 024002 (2001).

[3] C. R. Chen, J. L. Friar, and G. L. Payne, Few-Body Syst. 31, 13 (2001).

[4] S. Ishikawa, Few-Body Syst. 32, 229 (2003).

[5] A. Deltuva, A. C. Fonseca, and P. U. Sauer, Phys. Rev. C 71, 054005 (2005).

[6] E. O. Alt and M. Rauh, Few-Body Syst. 17, 121 (1994).

[7] A. Kievsky, M. Viviani, and S. Rosati, Phys. Rev. C 56, 2987 (1997).

[8] V. M. Suslov and B. Vlahovic, Phys. Rev. C 69, 044003 (2004).

[9] E. O. Alt, P. Grassberger, and W. Sandhas, Nucl. Phys. B2, 167 (1967).

[10] J. R. Taylor, Nuovo Cimento B 23, 313 (1974); M. D. Semon and J. R. Taylor, Nuovo Cimento A 26, 48 (1975).

[11] E. O. Alt, W. Sandhas, and H. Ziegelmann, Phys. Rev. C 17, 1981 (1978); E. O. Alt and W. Sandhas, ibid. 21, 1733 (1980).

[12] R. Machleidt, Phys. Rev. C 63, 024001 (2001).

[13] A. Deltuva, R. Machleidt, and P. U. Sauer, Phys. Rev. C 68, 024005 (2003).

[14] A. Deltuva, A. C. Fonseca, and P. U. Sauer, Phys. Rev. Lett. 95, 092301 (2005).

[15] A. Deltuva, K. Chmielewski, and P. U. Sauer, Phys. Rev. C 67, 034001 (2003).
[16] A. Deltuva, A. C. Fonseca, A. Kievsky, S. Rosati, P. U. Sauer, and M. Viviani, Phys. Rev. C 71, 064003 (2005).

[17] M. Yamaguchi, H. Kamada, and Y. Koike, nucl-th/0310024.

[18] A. Deltuva, K. Chmielewski, and P. U. Sauer, Phys. Rev. C 67, 054004 (2003).

[19] A. Deltuva, L. P. Yuan, J. Adam, Jr., A. C. Fonseca, and P. U. Sauer, Phys. Rev. C 69, 034004 (2004).

[20] A. Deltuva, L. P. Yuan, J. Adam, Jr., and P. U. Sauer, Phys. Rev. C 70, 034004 (2004).

[21] R. Großmann et al., Nucl. Phys. A603, 161 (1996).

[22] G. Rauprich et al., Nucl. Phys. A535, 313 (1991).

[23] H. Patberg, R. Grossman, G. Nitzsche, L. Sydow, S. Vohl, H. P. Schieck, J. Golak, H. Witala, W. Glockle, and D. Huber, Phys. Rev. C 53, 1497 (1996).

[24] J. Zejma et al., Phys. Rev. C 55, 42 (1997).

[25] J. Strate et al., Nucl. Phys. A501, 51 (1989).

[26] M. Allet et al., Phys. Rev. C 50, 602 (1994).

[27] M. Allet et al., Few-Body Syst. 20, 27 (1996).

[28] St. Kistryn et al., Phys. Rev. C 68, 054004 (2003); St. Kistryn et al., to be published, nucl-th/0508012.

[29] R. F. Carlson et al., Lett. Nuovo Cimento 8, 319 (1973).

[30] E. Stephan et al., AIP Conf. Proc. 768, 73 (2005).

[31] N. R. Kolb, P. N. Dezendorf, M. K. Brussel, B. B. Ritchie, and J. H. Smith, Phys. Rev. C 44, 37 (1991).

[32] W. Tornow et al., AIP Conf. Proc. 768, 138 (2005).

[33] K. Dow et al., Phys. Rev. Lett. 61, 1706 (1988).

[34] C. Marchand et al., Phys. Lett. B153, 29 (1985).

[35] R. S. Hicks et al., Phys. Rev. C 67, 064004 (2003). 